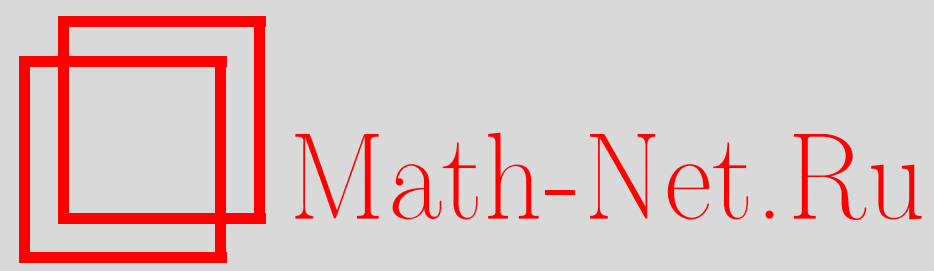

А. С. Кузьмин, Г. Б. Маршалко, Восстановление линейной рекурренты над примарным кольцом вычетов по ее усложнению. II, Матем. вопр. криптогр., 2011 , том 2, выпуск 2, 81-93

DOI: https://doi.org/10.4213/mvk32

Использование Общероссийского математического портала Math-Net.Ru подразумевает, что вы прочитали и согласны с пользовательским соглашением

http://www.mathnet.ru/rus/agreement

Параметры загрузки:

IP : 54.147 .182 .235

26 апреля 2023 г., 15:10:26 
УДК: $511.216,519.113 .6$

\title{
Восстановление линейной рекурренты над примарным кольцом вычетов по ее усложнению. II
}

\author{
А. С. Кузьмин ${ }^{1}$, Г. Б. Маршалко ${ }^{2}$ \\ ${ }^{1}$ Академия криптографии Российской Федерации, Москва \\ ${ }^{2}$ Лаборатория ТВП, Москва
}

Получено 22.IV.2010

Пусть $v-$ псевдослучайная последовательность над полем $\mathbb{Z}_{p}, p \geq 3$, которая получается усложнением знаков ЛРП $u$ максимального периода над кольцом $\mathbb{Z}_{p^{n}}$. В работе исследуются условия на преобразование, задающее усложнение рекурренты, при которых происходит сокращение периода последовательности .

Ключевые слова: Линейная рекуррентная последовательность, кольцо вычетов, примитивный многочлен

Reconstruction of linear recurrent sequence over prime residue ring from its image. II
A. S. Kuzmin ${ }^{1}$, G. B. Marshalko ${ }^{2}$
${ }^{1}$ Academy of Cryptography of Russian Federation, Moscow
${ }^{2}$ TVP Laboratory, Moscow

\begin{abstract}
Let $v$ be a pseudorandom sequence over $\mathbb{Z}_{p}, p \geq 3$, obtained from primitive sequence $u$ over the ring $\mathbb{Z}_{p^{n}}$ by means of some compressing map. We study conditions on the compressing map under which the period of $v$ is less than the period of the initial sequence $u$.

Key words: Compressing map, integer residue ring, linear recurrent sequence, primitive sequence

Citation: Mathematical Aspects of Cryptography, 2011, vol. 2, no. 2, pp. 81-93 (Russian).




\section{Введение}

Настоящая работа является продолжением исследований возможности восстановления линейной рекурренты (ЛРП) над кольцом вычетов $R=$ $=\mathbb{Z}_{p^{n}}, p>2$, по ее усложнению, начатых в [3].

В работе [3] исследовалась возможность восстановления ЛРП максимального периода над примарным кольцом вычетов по ее усложнению. При этом для некоторых классов функций усложнения были приведены алгоритмы восстановления начального вектора рекурренты и описаны условия, при которых не происходит сокращения периода усложненной последовательности по сравнению с периодом старшей координатной последовательности рекурренты.

В данной работе исследуются условия на преобразование, задающее усложнение рекурренты, при которых происходит сокращение периода усложненной последовательности.

\section{1. Математический аппарат}

Пусть $p$ - простое число, $n \in \mathbb{N}$ и $R=\left(\mathbb{Z}_{M},+, \cdot\right)-$ кольцо вычетов кольца $\mathbb{Z}$ целых чисел по модулю $M=p^{n}$ с единицей $e$. Пусть $F(x) \in R[x]-$ унитарный многочлен степени $m$ вида $F(x)=x^{m}-\sum_{j=0}^{m-1} f_{j} \cdot x^{j}$. Последовательность $u: \mathbb{N}_{0} \rightarrow R$ над кольцом $R$ называют линейной рекуррентной последовательностью порядка $m$ с характеристическим многочленом $F(x)$, если ее знаки удовлетворяют соотношениям

$$
u(i+m)=\sum_{j=0}^{m-1} f_{j} \cdot u(i+j), i=0,1,2, \ldots
$$

В этом случае унитарный многочлен $F(x)=x^{m}-\sum_{j=0}^{m-1} f_{j} \cdot x^{j}$ называют характеристическим многочленом последовательности $u$. Множество последовательностей с характеристическим многочленом $F(x)$ обозначим через $L_{R}(F)$.

Каждый элемент $a \in R$ имеет однозначное $p$-ичное представление вида

$$
a=\sum_{s=0}^{n-1} a_{s} \cdot p^{s}, \quad a_{s} \in \mathbb{Z}_{p}=\{0, \ldots, p-1\}, s \in 0, \ldots, n-1 .
$$


Будем называть коэффициент $a_{s}$ в последней сумме $s-\check{u}$ (р-ичной) координатой элемента $a$ и использовать обозначение $a_{s}=\delta_{s}(a)$. В соответствии с этим каждой последовательности $u \in R^{\infty}$ соответствуют $n$ последовательностей $u_{s}=\delta_{s}(u) \in \mathbb{Z}_{p}^{\infty}, \quad s \in 0, \ldots, n-1$, члены которых определяются соотношением

$$
u(i)=\sum_{s=0}^{n-1} u_{s}(i) \cdot p^{s}, \quad u_{s}(i)=\delta_{s}(u(i)), \quad s \in 0, \ldots, n-1 .
$$

Назовем $u_{0}, \ldots, u_{n-1}$ координатныли последовательностями последовательности $u$. Очевидно, каждая из этих последовательностей есть ЛРП над полем $\mathbb{Z}_{p}$.

Основным объектом изучения в качестве псевдослучайной последовательности (ПСП) являются последовательность $u_{n-1}$ и последовательности $v \in \mathbb{Z}_{p}^{\infty}$ более общего вида

$$
v(i)=\Psi(u(i)),
$$

где $\Psi: R^{n} \rightarrow \mathbb{Z}_{p}-$ некоторая функция. Будем кратко писать в этом случае $v=\Psi(u)$. Заметим, что, пользуясь разложением (1), функцию $\Psi(x)$ над $R$ можно рассматривать как многочлен $\Psi(x)=\psi\left(x_{0}, \ldots, x_{n-1}\right)$ над полем $\mathbb{Z}_{p}$ от переменных $x_{s}=\delta_{s}(x)$. При этом последовательности $z=u_{n-1}$ соответствует функция $\Psi(x)=\psi\left(x_{n-1}\right)=x_{n-1}$.

Для периода $T(F)$ любого многочлена $F(x) \in R[x]$ степени $m$ выполняется неравенство

$$
T(F) \leq p^{n-1}\left(p^{m}-1\right) .
$$

Если $T(F)=p^{n-1}\left(p^{m}-1\right)$, то многочлен $F$ называется многочленом максимального периода (МП-многочленом) или примитивным многочленом.

Любая ЛРП над $R$ ранга $m$ имеет период $T(u) \leq p^{n-1}\left(p^{m}-1\right)$. Последовательность $u$ над $R$ будем называть линейной рекуррентой максимального периода (ЛРП МП), если

$$
\operatorname{rk} u=m, \quad T(u)=p^{n-1}\left(p^{m}-1\right) .
$$

Назовем поле $\bar{R}=R / p R=\mathbb{Z}_{p}$ полем вычетов кольца $R$, и для любой последовательности $u \in R^{\infty}$ и любого многочлена $F(x) \in R[x]$ через $\bar{u}, \bar{F}$ обозначим соответствующие образы при естественном эпиморфизме $R \rightarrow \bar{R}$. Для любого унитарного многочлена $F(x) \in R[x]$ положим

$$
L_{R}(F)^{*}=\left\{u \in L_{R}(F): \bar{u} \neq \overline{0}\right\} .
$$


Тогда (3) равносильно тому, что для некоторого унитарного многочлена $F(x) \in R[x]$ степени $m$ выполняются условия

$$
u \in L_{R}(F)^{*}, \quad T(F)=p^{n-1}\left(p^{m}-1\right) .
$$

Заметим, что условие (4) влечет за собой условие $T(\bar{F}(x))=p^{m}-1$, т. е. $\bar{F}(x)$ - неприводимый и, более того, примитивный многочлен над $\bar{R}$.

В работах $[4,5,7,14]$ показано, что для многочлена максимального периода существуют многочлены $\Phi^{(s+1)}(x), s=0, \ldots, n-2$, такие, что справедливы следующие соотношения:

$$
\begin{aligned}
& x^{\tau_{s}}-e \equiv p^{s+1} \Phi^{(s+1)}(x)(\bmod F(x)), \operatorname{deg} \Phi^{(s+1)}(x)<m, \\
& \bar{\Phi}^{(s+1)}(x)=\bar{\Phi}^{(1)}(x) \neq \overline{0}, \quad s=0, \ldots, n-2,
\end{aligned}
$$

где $\tau_{s}=p^{s}\left(p^{m}-1\right)$.

Заметим, что многочлен $\Phi^{(s)}(x)$ определен однозначно только по модулю $p^{n-s}$.

В работах $[23,25]$ МП-многочлен $F(x)$ называется сильнопримитивнымм, если $\operatorname{deg}\left(\Phi^{(1)}(x)\right) \geq 1$.

Для любой последовательности ЛРП $u \in L_{R}(F)^{*}$ назовем последовательность

$$
u^{(s)}=\Phi_{s}(x) u
$$

s-ü производной (oт) последовательности и. Заметим, что в силу (5) справедливы равенства

$$
u_{0}^{(s)}=u_{0}^{(1)}, s=2, \ldots, n-1 .
$$

Следующая теорема описывает частоты появления обратимых элементов кольца на цикле рекурренты.

Теорема 1 ([14]). Пусть $F(x)-$ многочлен максимального периода степени $m, m \geq 2$, над примарным кольиом $R=\mathbb{Z}_{p^{n}}, p \geq 3, n \geq 2$. Тогда для частот $\nu_{u}(\alpha)$ появления элементов кольиа $R$ на цикле ЛРП МП $u \in L_{R}(F)$ справедливы следующие оценки:

если $F(x)$ не является сильнопримитивным, $m \geq 2 u \alpha$-обратимый элемент кольца $R$, то

$$
\begin{gathered}
\nu_{u}(\alpha)=p^{m-1}, \text { для } p \geq 3, \\
\nu_{u}(\alpha)=2^{m-2}, \text { для } p=2, n \geq 3
\end{gathered}
$$


если $F(x)$ - сильнопримитивньлй, $m \geq 3$, то

$$
\begin{aligned}
& \nu_{u}(\alpha) \geq(p-1) p^{m-2}, \text { для } p \geq 3 \\
& \nu_{u}(\alpha) \geq 2^{m-3}, \text { для } p=2, n \geq 3
\end{aligned}
$$

ЗАМЕЧАНИЕ 1. В частности, из теоремы 1 следует, что в любой ЛРП МП порядка $m \geq 3$ встречаются все обратимые элементы кольца $R$.

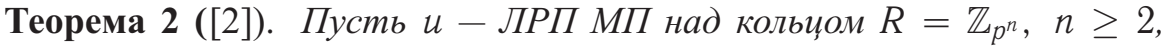
с характеристическим многочленом $F(x)$ степени $m \geq 4 n-2$, тогда в ЛРП и встречаются все элементы кольияа $R$.

Далее там, где возможно двусмысленное толкование, операции сложения, вычитания и умножения по модулю $p$ на координатном множестве $\mathbb{Z}_{p}$ обозначаются символами $\oplus, \ominus, \otimes$. В соответствии с этим произведение многочлена $\bar{A}(x) \in \bar{R}[x]$ и многочлена $f(x) \in \mathbb{Z}_{p}[x]$ на последовательность $u \in \mathbb{Z}_{p}^{\infty}$ обозначается, соответственно, $\bar{A}(x) \otimes u$ и $f(x) \otimes u$. Заметим, что $\bar{A}(x) \otimes u=\overline{A(x) u}$.

В работе [3] доказаны следующие две леммы о соотношениях между элементами координатных последовательностей ЛРП МП над примарными кольцами вычетов.

Лемма 1 ([3]). Для координатных последовательностей ЛРП МП и над кольцим $Z_{p^{n}}, p \geq 3$, справедливы следуюшие соотношения:

a) $n$ pu $s \in\{1, \ldots, n-1\}, j \in\{1, \ldots, p-1\}$

$$
\left(x^{j \tau_{s-1}}-e\right) \otimes u_{s}=j u_{0}^{(1)}
$$

b) при $s \in\{2, \ldots, n-1\} u \Delta(x, y)=\left\{\begin{array}{l}0, \text { если } x+y<p \\ 1, \text { если } x+y \geq p\end{array}\right.$

$$
\left(x^{\tau_{s-2}}-e\right) \otimes u_{s}=u_{1}^{(s-1)} \oplus \Delta\left(u_{s-1} ; u_{0}^{(1)}\right) ;
$$

c) при $0 \leq t<s-1$

$$
v_{s, t}=\left(u_{0}^{(1)}\right)^{p-1} \otimes u_{t+1} \oplus \frac{\left(u_{0}^{(1)}\right)^{p-1} \ominus u_{0}^{(1)}}{2} \oplus \xi(p, s, t),
$$

где

$$
\xi(p, s, t)= \begin{cases}\left(u_{0}^{(1)}\right)^{p-1} \otimes\left(\bar{\Phi}^{(1)}(x)^{2} \otimes u_{0}\right), & \text { если } p=3, t=0, s>2 \\ \bar{\Phi}^{(1)}(x)^{2} \otimes u_{0}, & \text { если } p=3, t=0, s=2 \\ 0 & \text { в остальных случаях }\end{cases}
$$


d) $n$ ри $s \in\{1, \ldots, n-1\}$

$$
\left(x^{\tau_{s-1}}-e\right)^{k} \otimes u_{s}^{k}=k !\left(u_{0}^{(1)}\right)^{k} ;
$$

e) $n p u s \in\{2, \ldots, n-1\}$

$$
\left(x^{\tau_{s-1}}-e\right)^{k-1} \otimes u_{s}^{k}=k !\left(u_{0}^{(1)}\right)^{k-1} u_{s} \oplus \frac{k-1}{2} k !\left(u_{0}^{(1)}\right)^{k} .
$$

Лемма 2 ([3]). Для координатных последовательностей ЛРП максимального периода справедливо следующее соотномение:

$$
u_{s}\left(i+\frac{\tau_{s}}{2}\right)=\left\{\begin{array}{l}
-u_{s}(i), \text { если } и(i) \equiv 0 \quad\left(\bmod p^{s}\right), \\
-e-u_{s}(i), \text { если } u(i) \not \equiv 0\left(\bmod p^{s}\right),
\end{array}\right.
$$

где $i \in\{0,1,2, \ldots\}, s \in\{1, \ldots, n-1\}$.

\section{2. Период усложненной рекурренты}

Теорема 3. Пусть $F(x)$ - многочлен максимального периода степени $m, m \geq 2$, над примарным кольцом $R=\mathbb{Z}_{p^{n}}, p \geq 3, n \geq 2, u-Л Р П$ МП из $L_{R}(F)$, v - последовательность, полученная усложнением последовательности $u$, такая, что $v(i)=\psi\left(u_{n-1}(i)\right), i \geq 0$, где $\psi-$ отличная от константы функиия. Тогда период $T(v)$ последовательности v удовлетворяет неравенству $T(v) \geq \frac{1}{2} T\left(u_{n-1}\right)$, и для выполнения равенства $T(v)=\frac{1}{2} T\left(u_{n-1}\right)$ необходимо, чтобы выполнялись следующие условия:

(a) многочлен $F(x)$ не является сильнопримитивным;

(b) $\psi(x \ominus e)=\psi(-x)$;

(c) в ЛРП и отсутствуют элементы вида $p^{n-1} \varepsilon, \varepsilon \not \equiv 0(\bmod p)$.

Доказательство. Пусть $t=T(v)-$ период последовательности $v=$ $=\psi\left(u_{n-1}\right)$. При этом очевидно, что $t \mid \tau_{n-1}$. Будем считать далее, что функция $\psi(x)$ имеет вид

$$
\psi\left(x_{n-1}\right)=\sum_{j=0}^{k} c_{j} x_{n-1}^{j}, \quad 0<k<p, \quad c_{k} \neq 0 .
$$

Тогда с учетом леммы 2 имеем

$$
\left(x^{\tau_{n-2}} \ominus e\right)^{k} \otimes \psi\left(u_{n-1}\right)=c_{k} k !\left(u_{0}^{(1)}\right)^{k} .
$$


Так как период последовательности $\left(x^{\tau_{n-2}} \ominus e\right)^{k} \otimes \psi\left(u_{n-1}\right)$ делит $t$, а период последовательности $\left(u_{0}^{(1)}\right)^{k}$ делится на $\frac{p^{m}-1}{p-1}$, то

$$
\frac{p^{m}-1}{p-1} \mid t
$$

С другой стороны, из (14) следует, что $p^{n-1} \mid t$. Таким образом, получаем, что период $t$ последовательности $v$ представляется в виде

$$
t=\frac{p^{m}-1}{p-1} p^{n-1} l, \text { где } l \mid p-1
$$

Рассмотрим, при каких условиях может выполняться неравенство $l<$ $<p-1$. Из п. d) и е) леммы 1 вытекает равенство

$$
\begin{gathered}
\left(x^{\tau_{n-2}} \ominus e\right)^{k-1} \otimes \psi\left(u_{n-1}\right)=c_{k} k !\left(u_{0}^{(1)}\right)^{k-1} u_{n-1} \oplus \\
\oplus c_{k} \frac{k-1}{2} k !\left(u_{0}^{(1)}\right)^{k} \oplus c_{k-1}(k-1) !\left(u_{0}^{(1)}\right)^{k-1} .
\end{gathered}
$$

Учитывая, что период последовательности $\left(x^{\tau_{n-2}} \ominus e\right)^{k-1} \otimes \psi\left(u_{n-1}\right)$ делит $t$, для $i \geq 0$ получаем

$$
\begin{gathered}
c_{k} k !\left(u_{0}^{(1)}(i+t)\right)^{k-1} u_{n-1}(i+t) \oplus c_{k} \frac{k-1}{2} k !\left(u_{0}^{(1)}(i+t)\right)^{k} \oplus \\
\oplus c_{k-1}(k-1) !\left(u_{0}^{(1)}(i+t)\right)^{k-1}=c_{k} k !\left(u_{0}^{(1)}(i)\right)^{k-1} u_{n-1}(i) \oplus \\
\oplus c_{k} \frac{k-1}{2} k !\left(u_{0}^{(1)}(i)\right)^{k} \oplus c_{k}(k-1) !\left(u_{0}^{(1)}(i)\right)^{k-1} \cdot
\end{gathered}
$$

В силу свойств мультипликаторов ЛРП МП над конечными полями [18] существует примитивный элемент $\alpha \in G F(p)$ такой, что

$$
u_{0}^{(1)}\left(i+\frac{p^{m}-1}{p-1} p^{n-1} l\right)=\alpha^{l} u_{0}^{(1)}(i), \quad i \geq 0 \text {. }
$$

Подставляя (16) в (15), получаем

$$
\begin{gathered}
c_{k} k !\left(u_{0}^{(1)}(i)\right)^{k-1}\left(u_{n-1}(i+t) \alpha^{(k-1) l} \ominus u_{n-1}(i)\right)= \\
=c_{k} \frac{k-1}{2} k !\left(u_{0}^{(1)}(i)\right)^{k}\left(e \ominus \alpha^{k l}\right) \oplus c_{k-1}(k-1) !\left(u_{0}^{(1)}(i)\right)^{k-1}\left(e \ominus \alpha^{(k-1) l}\right) .
\end{gathered}
$$


Из (14) следует, что $t$ делится на период последовательности $\left(u_{0}^{(1)}\right)^{k}$. С другой стороны, справедливо равенство

$$
\left(u_{0}^{(1)}(i+t)\right)^{k}=\alpha^{k l}\left(u_{0}^{(1)}(i)\right)^{k}, \quad i \geq 0 .
$$

Следовательно, $\alpha^{k l}=e$, и соотношение (17) приобретает вид

$$
\begin{gathered}
c_{k} k !\left(u_{0}^{(1)}(i)\right)^{k-1}\left(u_{n-1}(i+t) \alpha^{-l} \ominus u_{n-1}(i)\right)= \\
=c_{k-1}(k-1) !\left(u_{0}^{(1)}(i)\right)^{k-1}\left(e \ominus \alpha^{-l}\right) .
\end{gathered}
$$

Выберем $i$ таким, что $u_{0}^{(1)}(i) \neq 0$, тогда

$$
u_{n-1}(i+t)=\alpha^{l}\left(u_{n-1}(i) \oplus \frac{c_{k-1}}{c_{k} k}\left(e \ominus \alpha^{-l}\right)\right)
$$

Из представления ЛРП МП над примарными кольцами вычетов через функцию след (см. $[6,11,14])$ и из $(16)$ вытекает, что существует элемент $\tilde{\alpha} \in R$ со свойствами

$$
\begin{aligned}
& \tilde{\alpha} \equiv \alpha(\bmod p), \quad \tilde{\alpha}^{p-1}=e, \\
& u\left(i+\frac{\tau_{n-1}}{p-1}\right)=\tilde{\alpha} u(i), \quad i \geq 0 .
\end{aligned}
$$

Отсюда вытекает, что для $i \geq 0$ имеют место равенства

$$
u_{n-1}(i+t) \ominus \alpha^{l} u_{n-1}(i)=\eta\left(\tilde{\alpha}, u_{0}(i), \ldots, u_{n-2}(i)\right),
$$

где $\eta\left(\tilde{\alpha}, u_{0}(i), \ldots, u_{n-2}(i)\right)-$ функция, равная $(n-1)$-му разряду $p$-ичного разложения элемента $\tilde{\alpha}^{l} \sum_{j=0}^{n-2} u_{j}(i) p^{j}$. Согласно (19), во всех тактах $i$, когда $u_{0}^{(1)}(i) \neq 0$, должно выполняться равенство

$$
\eta\left(\tilde{\alpha}, u_{0}(i), \ldots, u_{n-2}(i)\right)=\alpha^{l} \frac{c_{k-1}}{c_{k} k}\left(e \ominus \alpha^{-l}\right) .
$$

Если многочлен $F(x)$ сильнопримитивный, то в тех тактах, когда $u_{0}^{(1)}(i) \neq 0$, на цикле ЛРП МП $u$ встречаются все элементы кольца $R$ (см. теорему 1). В частности, для некоторых $j_{0}$ и $j_{1}$ будут выполнены условия

$$
\begin{array}{cc}
u_{0}^{(1)}\left(j_{0}\right) \neq 0, & u_{0}^{(1)}\left(j_{1}\right) \neq 0, \\
u\left(j_{0}\right) \equiv 0 \quad\left(\bmod p^{n-1}\right), \\
u\left(j_{1}\right) \equiv \beta \quad\left(\bmod p^{n-1}\right),
\end{array}
$$


где $\beta$ - наименьшее число из $1,2, \ldots, p^{n-1}-1$ со свойством $\eta\left(\tilde{\alpha}, u_{0}(i), \ldots, u_{n-2}(i)\right) \neq 0$. Такое $\beta$ существует, так как $\tilde{\alpha}^{l} \neq e$. Для этих $j_{0}$ и $j_{1}$

$$
\begin{aligned}
& \eta\left(\tilde{\alpha}, u_{0}\left(j_{0}\right), \ldots, u_{n-2}\left(j_{0}\right)\right)=0 \\
& \eta\left(\tilde{\alpha}, u_{0}\left(j_{1}\right), \ldots, u_{n-2}\left(j_{1}\right)\right) \neq 0 .
\end{aligned}
$$

В результате мы пришли к противоречию с (19). Следовательно, если $F(x)$ является сильнопримитивным, то период последовательности $v$ равен периоду ЛРП $u_{n-1}$.

Если многочлен $F(x)$ не является сильнопримитивным, то в тех тактах, когда $u_{0}^{(1)}(i) \neq 0$, на цикле ЛРП МП $u$ встречаются все обратимые элементы кольца $R$ (теорема 1). Из (20) следует, что

$$
\eta(\tilde{\alpha}, e, 0, \ldots, 0)=\eta(\tilde{\alpha}, 2 e, 0, \ldots, 0) .
$$

получаем

$\mathrm{C}$ другой стороны, по определению функции $\eta\left(\tilde{\alpha}, \beta_{0}, \ldots, \beta_{n-2}\right)$

$$
\eta(\tilde{\alpha}, 2 e, 0, \ldots, 0)=2 \eta(\tilde{\alpha}, e, 0, \ldots, 0) \oplus \Delta_{n-1}\left(\tilde{\alpha}^{l}, \tilde{\alpha}^{l}\right),
$$

где $\Delta_{n-1}(x, y)$ - функция переноса в $(n-1)$-й разряд от сложения чисел $x$ и $y$ в $p$-ичной системе счисления. Поэтому $\eta(\tilde{\alpha}, e, 0, \ldots, 0) \in$ $\in\{0, p-1\}$. Если $\eta(\tilde{\alpha}, e, 0, \ldots, 0)=0$, то находим наименьшее $\beta$ со свойством $\eta\left(\tilde{\alpha}, \beta_{0}, \ldots, \beta_{n-2}\right) \neq 0$. Очевидно, что $\eta\left(\tilde{\alpha}, \beta_{0}, \ldots, \beta_{n-2}\right)=e$. Тогда $\eta\left(\tilde{\alpha}, \beta_{0}+e, \ldots, \beta_{n-2}\right) \in\{1,2\}$, и в силу условия $p \geq 3$ значение $\eta\left(\tilde{\alpha}, \beta_{0}+e, \ldots, \beta_{n-2}\right)$ отлично от нуля. При этом хотя бы один из элементов $\beta$ или $\beta+e$ обратим в $R$, поэтому существует номер $i$, когда $u_{0}^{(1)}(i) \neq 0$ и $u(i)$ является обратимым элементом. Получаем противоречие с (20) и, следовательно, $\eta(\tilde{\alpha}, e, 0, \ldots, 0)=p-1$.

Пусть элементы $x$ и $x+e$ обратимы в кольце $R$, тогда

$$
\begin{gathered}
\eta\left(\tilde{\alpha}, x_{0}+e, x_{1}, \ldots, x_{n-2}\right)=\Delta_{n-1}\left(\tilde{\alpha}^{l} x, \tilde{\alpha}^{l}\right) \oplus \\
\oplus \eta(\tilde{\alpha}, e, 0, \ldots, 0) \oplus \eta\left(\tilde{\alpha}, x_{0}, x_{1}, \ldots, x_{n-2}\right) .
\end{gathered}
$$

Отсюда следует, что

$$
\Delta_{n-1}\left(\tilde{\alpha}^{l} x, \tilde{\alpha}^{l}\right)=e
$$

для любого такого $x \in R$, что $x$ и $x+e$ обратимы в кольце $R$.

Пусть $\tilde{\alpha}^{l} \neq-e$, тогда $\Delta_{n-1}\left(\tilde{\alpha}^{l}, e\right)=0$, и, поскольку $\tilde{\alpha}^{-l}-$ корень уравнения $x^{p}=x$, элементы $\tilde{\alpha}^{-l}$ и $\tilde{\alpha}^{-l}+e$ обратимы в $R$. Приходим к противоречию с (21). 
Таким образом, неравенство $t<p^{n-1}\left(p^{m}-1\right)$ возможно только в случае

$$
\tilde{\alpha}^{l} \equiv-e \quad\left(\bmod p^{n-1}\right), \quad \eta(\tilde{\alpha}, e, 0, \ldots, 0)=p-1,
$$

т. е. в случае $\tilde{\alpha}^{l}=p^{n}-1$. При этом для любого обратимого элемента кольца $R$ имеет место соотношение

$$
\Delta_{n-1}\left(p^{n}-1, x\right)=e .
$$

В силу свойств элемента $\tilde{\alpha}$ равенство $\tilde{\alpha}^{l}=p^{n}-1$ эквивалентно условию $l=\frac{p-1}{2}$. Откуда следует, что период последовательности $v$ равен $t=\frac{\tau_{n-1}}{2}$.

Если $F(x)$ не является сильнопримитивным и $u_{0}^{(1)}(i) \neq 0$, то $u(i) \not \equiv$ $\not \equiv 0\left(\bmod p^{n-1}\right)$, тогда, используя лемму 2 , получаем

$$
u_{n-1}\left(i+\frac{\tau_{n-1}}{2}\right)=-u_{n-1}(i) \ominus e, \quad i \geq 0 .
$$

Так как $t=\frac{\tau_{n-1}}{2}-$ период последовательности $v=\psi(x)$, то

$$
\psi\left(u_{n-1}\left(i+\frac{\tau_{n-1}}{2}\right)\right)=\psi\left(-u_{n-1}(i) \ominus e\right)=\psi\left(u_{n-1}(i)\right) .
$$

В тактах с $u_{0}^{(1)} \neq 0$ в ЛРП $u_{n-1}$ встречаются все элементы поля $G F(p)$ (см. теорему 1), поэтому (22) эквивалентно равенству

$$
\psi(x \ominus e)=\psi(-x) .
$$

Предположим, что в ЛРП $u$ есть элементы вида $p^{n-1} \epsilon, \epsilon \not \equiv 0(\bmod p)$, т. е. для некоторого $i$ выполняется равенство $u(i)=p^{n-1} \epsilon$. Тогда в силу леммы 2 имеем

$$
u_{n-1}\left(i+\frac{\tau_{n-1}}{2}\right)=-u_{n-1}(i), \quad i \geq 0,
$$

и, учитывая, что $t=\frac{\tau_{n-1}}{2}-$ период последовательности $v$, получаем $\psi(\epsilon)=$ $=\psi(-\epsilon)$. При этом в силу свойств элемента $\tilde{\alpha}$ справедливо равенство

$$
u_{n-1}\left(i+j \frac{\tau_{n-1}}{p-1}\right)=\tilde{\alpha}^{j} p^{n-1} \epsilon, \quad j=1, \ldots, p-1,
$$

и из примитивности элемента $\alpha$ в поле $G F(p)$ вытекает, что в ЛРП $u_{n-1}$ встречаются все элементы вида $p^{n-1} \epsilon, \epsilon \in\{1,2, \ldots, p-1\}$. Следовательно, равенство $\psi(x)=\psi(-x)$ выполняется для всех ненулевых элементов из $G F(p)$. Отсюда с учетом (23) следует, что

$$
\psi(x)=\text { const },
$$

а это противоречит условию теоремы. 
Таким образом, сокращение периода ЛРП $u_{n-1}$ при применении функции усложнения $\psi(x)$ возможно только при одновременном выполнении условий:

$$
\begin{aligned}
& \operatorname{deg} \overline{\Phi_{1}}(x)=0, \\
& \psi(x \ominus e)=\psi(-x), \\
& \text { в ЛРП } u \text { отсутствуют элементы вида } p^{n-1} \varepsilon, \varepsilon \not \equiv 0(\bmod p) .
\end{aligned}
$$

При этом сокращение периода по сравнению с периодом ЛРП $u_{n-1}$ будет не более чем в 2 раза.

Следствие 1. Если в условиях теоремы 3 многочлен $F(x)$ является сильнопримитивным, то при любой отличной от константы функиии усложнения период $T(v)$ последовательности v равен $p^{n-1}\left(p^{m}-1\right)$.

Следствие 2. Если в условиях теоремы 3 функция $\psi(x)$ удовлетворяет условию $\psi(x \ominus e) \neq \psi(-x)$, то период $T(v)$ последовательности v равен $p^{n-1}\left(p^{m}-1\right)$.

Следствие 3. Если в условиях теоремы 3 степень многочлена $F(x)$ удовлетворяет неравенству $m \geq 4 n-2$, то при любой отличной от константы функции усложнения и любом многочлене $F(x)$ период $T(v)$ последовательности г равен $p^{n-1}\left(p^{m}-1\right)$.

Доказательство. Из теоремы 2 следует, что при $m \geq 4 n-2$ в ЛРП МП $u \in L_{R}(F)$ встречаются все ненулевые элементы кольца $R$, далее, пользуясь теоремой 3 , получаем требуемое утверждение.

\section{Список литературы}

1. Ван дер Варден Б. Л. Алгебра. - М.: Наука, 1979.

2. Камловский О.В., Кузьмин А.С. Оценки частот появления элементов в линейных рекуррентных последовательностях над кольцами Галуа. Фундам. и прикл. матем., 2000, т. 6, вып. 4, с. 1083-1094.

3. Кузьмин А.С., Маршалко Г.Б., Нечаев А.А. Восстановление линейной рекурренты над примарным кольцом вычетов по ее усложнению. Математические вопросы криптографии, 2010, т. 1, вып. 2, с. 31-56.

4. Кузьмин А.С., Нечаев А.А. Построение помехоустойчивых кодов с использованием линейных рекуррент над кольцами Галуа. - Успехи матем. наук, 1992, т. 47, вып. 5, с. 183-184.

5. Kuzmin A.S., Nechaev A.A. Linear recurrent sequences over Galois rings. In: Proc. $2^{\text {nd }}$ Int. Conf. Algebra, Barnaul, 1991. 
6. Нечаев А. А. Код Кердока в циклической форме. - Дискрет. матем., 1989, т. 1, № 4, с. 123-139.

7. Кузьмин А. С., Нечаев А. А. Линейные рекуррентные последовательности над кольцами Галуа. - Алгебра и логика, 1995, т. 3, № 2, с. 169-189.

8. Кузьмин А.С., Куракин В.Л., Нечаев А.А. Псевдослучайные и полилинейные последовательности. - В сб.: Труды по дискретной математике. Т. 1. - М.: ТВП, 1997, с. 139-202.

9. Кузьмин А.С., Куракин В.Л., Нечаев А. А. Свойства линейных и полилинейных рекуррент над кольцами Галуа. - В сб.: Труды по дискретной математике. Т. 2. - М.: ТВП, 1997, с. 191-222.

10. Кузьмин А. С., Куракин В.Л., Нечаев А. А. Свойства линейных и полилинейных рекуррент над кольцами Галуа (II). - Обозр. прикл. и промышл. матем., 2000, т. 7, вып. 1, с. 5-59.

11. Кузьмин А.С., Куракин В.Л., Нечаев А.А. Структурные аналитические и статистические свойства линейных и полилинейных рекуррент. - В сб.: Труды по дискретной математике. Т. 3. - М.: ТВП, 2000, с. 155-194.

12. Кузьлин А.С., Куракин В.Л., Нечаев А.А. Вполне равномерные линейные рекурренты. - В сб.: Труды по дискретной математике. Т. 5. - М.: ФИЗМАТЛИТ, 2002, с. 103-158.

13. Нечаев А. А. Линейные рекуррентные последовательности над коммутативными кольцами. - Дискрет. матем., 1991, т. 3, № 4, с. 107-121.

14. Kurakin V.L., Kuzmin A.S., Mikhalev A.V., Nechaev A.A. Linear recurring sequences over rings and modules. - J. Math. Sci., 1995, v. 76, № 6, p. $2793-$ 2915.

15. Кузьмин А. С., Нечаев А. А. Линейные рекуррентные последовательности над кольцами Галуа. - Успехи матем. наук, 1993, т. 48, вып. 1, с. 167-168.

16. Кузьмин А.С. Нижние оценки рангов координатных последовательностей линейных рекуррентных последовательностей над примарными кольцами вычетов. - Успехи матем. наук, 1993, т. 48, вып. 3, с. 193-194.

17. Кузьмин А.С. Распределение элементов на циклах линейных рекуррент над кольцами вычетов - Успехи матем. наук, 1992, т. 47, вып. 6, с. $213-$ 214.

18. Лидл Р., Нидеррайтер Г. Конечные поля, тт. 1, 2. - М.: Мир., 1988, 824 с.

19. Laxton R.R., Anderson J.A. Linear recurrences and maximal length sequences. - Math. Gazette, 1972, v. 56, p. 299-309.

20. Min-Qiang Huang, Zong-Duo Dai. Projective maps of linear recurring sequences with maximal $p$-adic periods. - Fibonacchi Quart., 1992, v.30, № 2, p. 139-143. 
21. Min-Qiang Huang. Analysis and cryptologic evaluation for primitive sequences over an integer residue ring. - Ph.D. diss., Graduate School of USTC, Acad. Sinica, Beijing, China, 1988.

22. Xuan-Yong Zhu, Weng-Feng Qi. Uniqueness of the distribution of zeros of primitive level sequences over $Z_{p^{n}}$. Finite Fields Appl., 2005, v. 11(1), p. 30 44.

23. Xuan-Yong Zhu, Weng-Feng Qi. Compression mappings on primitive sequences over $Z_{p^{n}}$. - IEEE Trans. Inf. Theory, 2004, v. 50, № 10, p. 2442-2448.

24. Xuan-Yong Zhu, Wen-Feng Qi. Further result of compressing maps on primitive sequences modulo odd prime powers. - IEEE Trans. Inf. Theory, 2007, v. 53, № 8, p. 2985-2990.

25. Tian Tian, Wen-Feng Qi. Injectivity of compressing maps on primitive sequences over $\mathbb{Z} /\left(p^{e}\right)$. - IEEE Trans. Inf. Theory, 2007, v. 53, № 8 , p. 2960-2966.

26. Zong-Duo Dai. Binary sequences derived from ML-sequences over rings. I: periods and minimal polynomials. - J. Cryptology, 1992, v. 5, № 4, p. 193207.

27. Weng-Feng Qi, Xuan-Yong Zhu. Compressing maps on primitive sequences over $Z_{2^{n}}$ and its Galois extension. - Finite Fields Appl., 2002, v. 8, № 4, p. 570-588.

28. Weng-Feng Qi. Compressing maps on primitive sequences over $Z_{2^{n}}$ and analysis of their derivate sequences. - Ph. D. diss., Zhengzhou Inform. Eng. Univ., 1997, Zhengzhou, China.

29. Weng-Feng Qi, Jun-Hui Yang, Jin-Jun Zhou. ML-sequences over rings $Z_{2^{n}}$. ASIACRYPT'98, Lect. Notes Comput. Sci., 1998, v. 1514, p. 315-325. 